\title{
Characterization of a combustion process by measurements of the spatial IR radiation of the flame
}

\author{
by Carlos Toro*, Luis Arias** and Sergio Torres***
}

*Dept. of Electrical Engineering, University of Concepción, Casilla 160-C Concepción, Chile, ctoron@udec.cl

** Dept. of Electrical Engineering, University of Concepción, Casilla 160-C Concepción, Chile, luiarias@udec.cl

${ }^{* * * D e p t . ~ o f ~ E l e c t r i c a l ~ E n g i n e e r i n g, ~ U n i v e r s i t y ~ o f ~ C o n c e p c i o ́ n, ~ C a s i l l a ~ 160-C ~ C o n c e p c i o ́ n, ~ C h i l e, ~}$ sertorre@udec.cl

\begin{abstract}
In this paper we characterize a liquefied gas flame by means of infrared image processing aimed to obtain variables that relates the infrared digital data with the combustion process. An infrared camera that capture radiation between $8[\mu \mathrm{m}]$ to $12[\mu \mathrm{m}]$ was used, different techniques as the histogram and FFT (fast Fourier transform) were calculated from infrared images and used for the data processing of different variables like axial and radial profiles, radiative zones, peaks of radiation and a frequency analysis of the reaction zone under different combustion conditions. The experimental results have shown a high correlation between the analyzed variables and the air rate conditions making the infrared radiation analysis an important tool for the flame sensing.
\end{abstract}

\section{Introduction}

Thermal radiation is produced by any body with an absolute temperature higher than $0[\mathrm{~K}]$. Thus, an infrared camera is capable to detect this energy using a spatial array of sensors in the focal plane and turning into an image. Nowadays, this information has been strongly used for medical diagnosis, material characterization, military applications, night vision, building insulation, detection of refrigerant leaks and the evaluation of many processes emitting in the IR (infrared) band. In this context, optical methods have been widely used for flame sensing and combustion process monitoring [1-5]. The aim of these studies is to find some meaningful characteristics of the acquired IR signals to correlate them with some combustion parameters or variables as the air-fuel rate, combustion efficiency, pollutant emissions, flame stability, etc, for the development of control strategies to generate more efficient, flexible, reliable and clean combustion systems.

In this study we characterize a low power flame of liquefied petroleum gas (LPG) generated in a Bunsen-type burner, in function of different air-rates mixed with the LPG. This task has been tackled by calculating spatial (axial and radial) emitting profiles, the histogram of different radiative zones and the spatial variation of the peaks associated to the reaction zone of the flame. A frequency analysis over the temporal variation of different isothermal zones of the flame is conducted where some features can be obtained. A frequency analysis pointing in the estimation of the flicker frequency has been considered, due to this indicator has been strongly used for flame stability analysis [7-8].

This work is organized as follows. In section 2, the description of the theory and the parameters involved in the processing are presented. In section 3 the experimental set-up and the methods used for acquiring and processing the data are described. In section 4 some results are showed and finally in section 5 some conclusions extracted from the analysis are summarized.

\section{Theoretical background}

A digital image of a flame is a two-dimensional projection of a three-dimensional flow field, where the gray-level of each pixel represents the luminous intensity (for the visible band) or thermal intensity (for the IR band). Fig. 1 shows flame images in the visible (VIS) and IR bands.

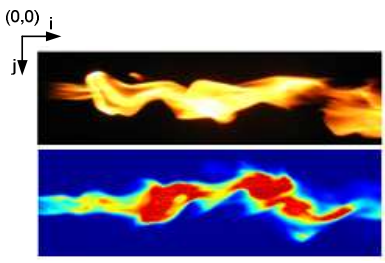

Figure 1. Flame images at the same air rate condition in the visible (upper) and infrared (lower) bands. 
The pseudocolor map used in the IR images shows the hottest regions as reddish and the coolest as bluish, also the IR camera doesn't give absolute temperature values without an offline radiometric calibration, each image will be consider as the equivalent thermal emittance radiated from the flame, $E$, which is related to the superficial temperature of the flame according to the Planck's Radiation Law.

Based on previous studies of flame images in the visible band $[7,8]$ we estimate the following spatial and spectral parameters from the IR images:

- Radial profile: maximum thermal emittance values across the flame

- Axial profile: maximum thermal emittance values along the flame

- Radiative power distribution: this analysis is made by using Histogram analysis in different regions of the flame

The flame stability is calculated by means of spectral analysis using the FFT algorithm and calculating the flickering frequency of a specific dynamic signal acquired from the flame. In our case, the hottest regions of the flames are studied. The quantitative flickering frequency is determined as the power spectral density (PSD) weighted average frequency over the entire frequency range. This variable is calculated as follow:

$$
F=\frac{\sum_{i}^{N} p_{i} f_{i}}{\sum_{i=1}^{N} p_{i}}
$$

where $F$ is the flickering frequency, $f_{i}$ is the ith frequency, $p_{i}$ is the power spectral density of the ith frequency component and $N$ is the number of frequency components.

\section{Experimental setup and methodology}

The experimental setup was carrying out by using a Bunsen-type burner of triple out, with LPG fuel. A Jade Uncooled (UC) IR camera (CEDIP) was used for flame sensing. Some specifications of the camera are summarized in Table 1.

Table 1. Technical specifications of the IR camera

\begin{tabular}{|l|l|}
\hline Model & Jade UC \\
\hline Detector type & $\begin{array}{l}\text { Focal plane Array, uncooled } \\
\text { microbolometer } 320(\mathrm{H}) \times 240(\mathrm{~V})\end{array}$ \\
\hline Spectral range & $8-12[\mu \mathrm{m}]$ \\
\hline Frame rate & $60[\mathrm{~Hz}]$ \\
\hline Digital output & $14[\mathrm{bit}]$, USB \\
\hline
\end{tabular}

The IR camera was placed $2[\mathrm{~m}]$ from the flame and for each of the ten air rate values, regulated in the base of the burner from 0[\%] to 100[\%], 100 frames were recorded with a sample frequency of $F_{s}=60[\mathrm{~Hz}]$. The images were processed in Matlab where each pixel was normalized by the higher digital level extracted from the data.

For the spatial analysis, the average of the whole frames at each measurement is calculated in order to smooth the flame turbulence. We refer to them as the average emittance images, $E_{\mathrm{av}}$.

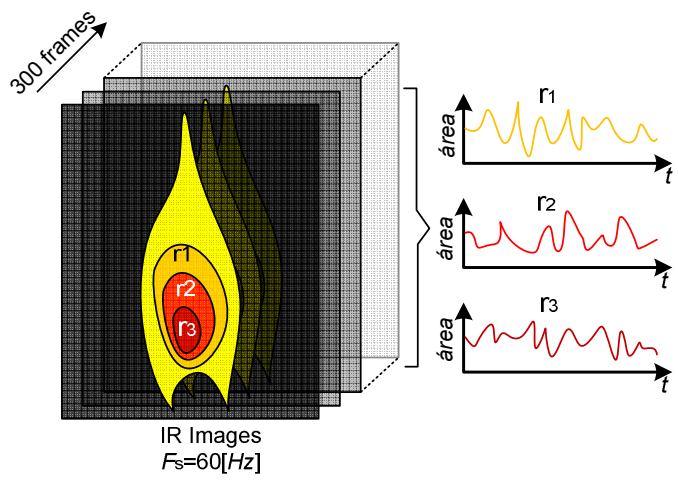

Figure 2. Processing scheme for the three different radiative regions analyzed. 
The frequency analysis uses all frames from each measurement without averaging. In this analysis we select three different intensity regions $\left(r_{1}\right.$ to $\left.r_{3}\right)$ from each flame to apply the Fourier analysis, $\left.\left.\left.\left.r_{1}=[0.7-0.8], r_{2}=\right] 0.8-0.9\right], r_{3}=\right] 0.9-1.0\right]$ obtaining 3 signals with 100 samples representing the temporal dynamics of these regions. It can be seen in Fig. 2.

\section{Experimental results}

In this section are shown the experimental results obtained by carrying out both, a spatial characterization of the IR flame radiation and the axial and radial profiles analysis considering different combustion conditions.

\subsection{Spatial characterization of flame by using IR images}

Fig. 3 shows the infrared averaged images of the flames for the complete set of data, considering different air rates from $100 \%$ to $0 \%$. It can be seen that for higher air rates, the flames become smaller and wider, the opposite situation to the ones produced by small air rates. The explanation to this refers to the need of the fuel excess to find the required oxidant to combust. Other interesting fact is that we can see the gases released for the flame (clear bluish colors) which are not observable in the visible spectrum.

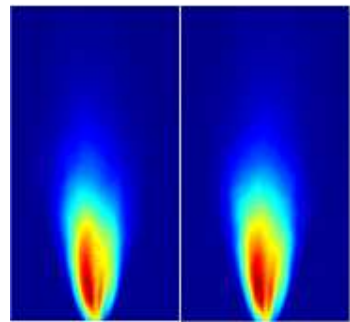

(a) (b)

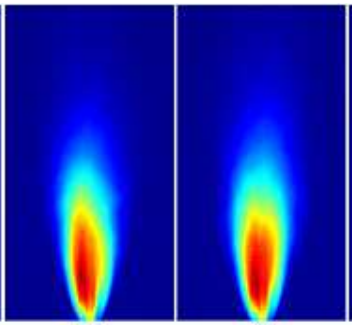

(c) (d)

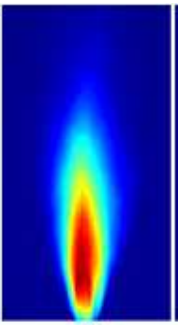

(e)

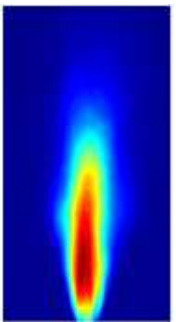

(f)

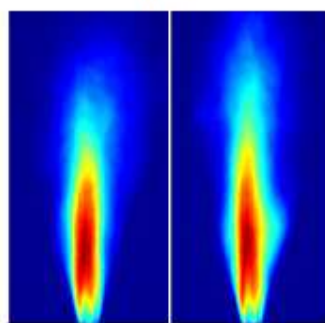

(g)

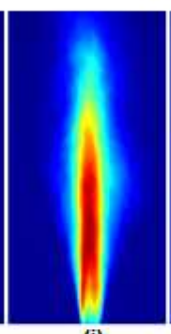

(i)

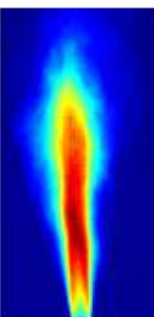

(j)

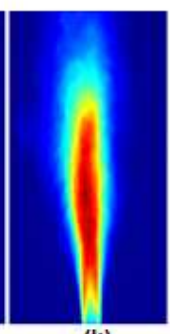

(k)

Figure. 3. Averaged frames of each measurement, for air-rates at (a) $100 \%$, (b) $90 \%$, (c) $80 \%$, (d) $70 \%$, (e) $60 \%$, (f) $50 \%$, (g) $40 \%$, (h) $30 \%$, (i) $20 \%$, (j) $10 \%$, (k) $0 \%$.

\subsection{Axial and radial radiative profiles}

In this analysis we consider the maximum axial and radial profiles on the flame. In Fig. 4 it is shown the profiles for each measurement as a function of the air-rate. In Fig. 5 the peak values of the profiles according to their position on the flame image are shown. It can be seen the hottest spots moving from the lower positions on the flame, near the burner exit, to higher positions when the air rate decreases.

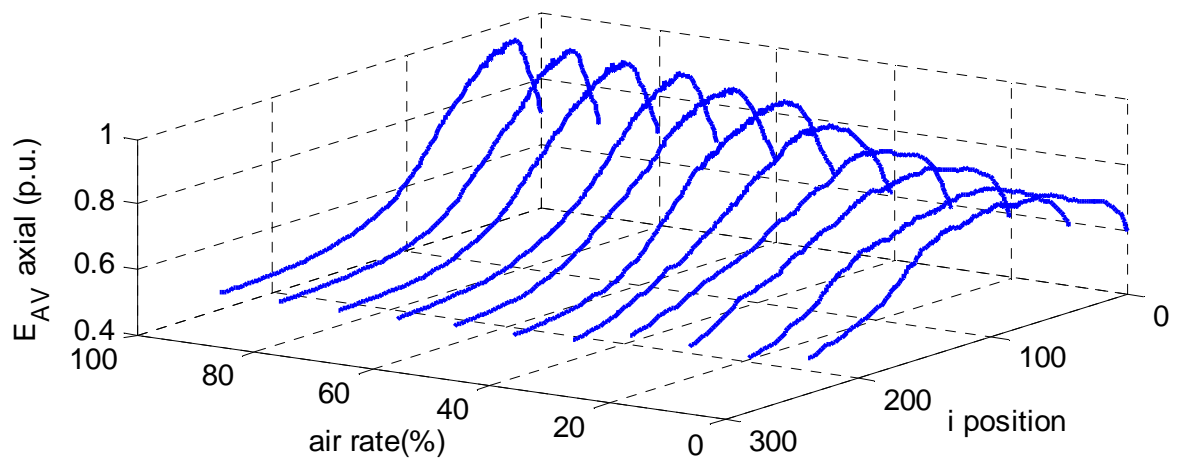

(a) 


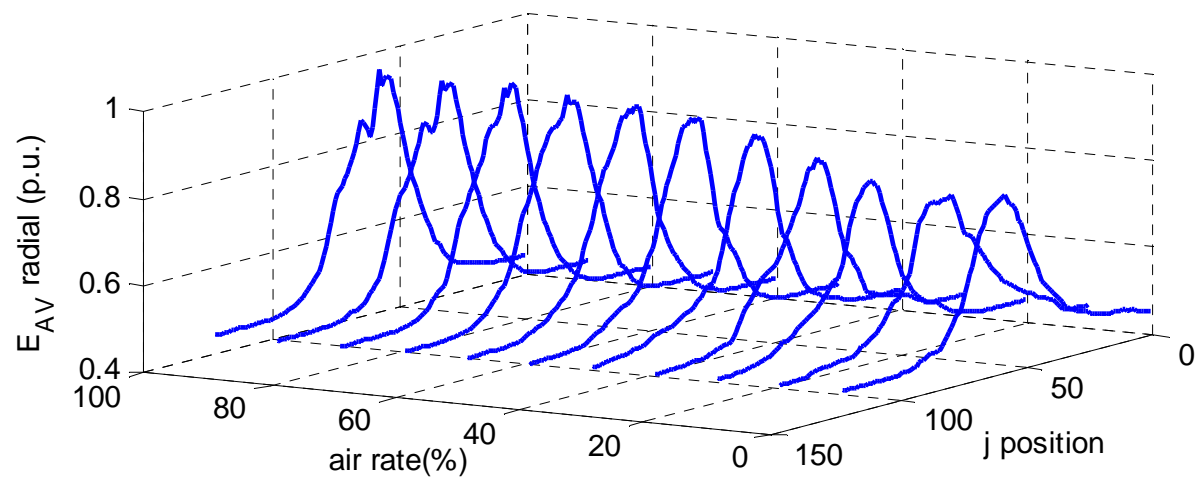

(b)

Figure 4. Axial and radial radiative profiles of the averaged frames for each measurement.

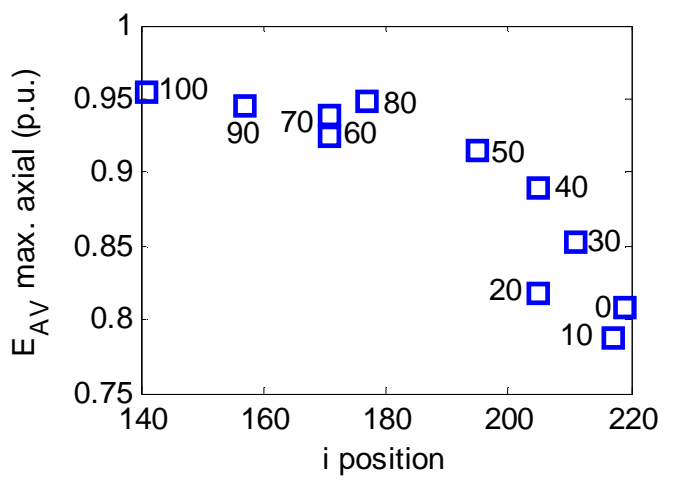

(a)

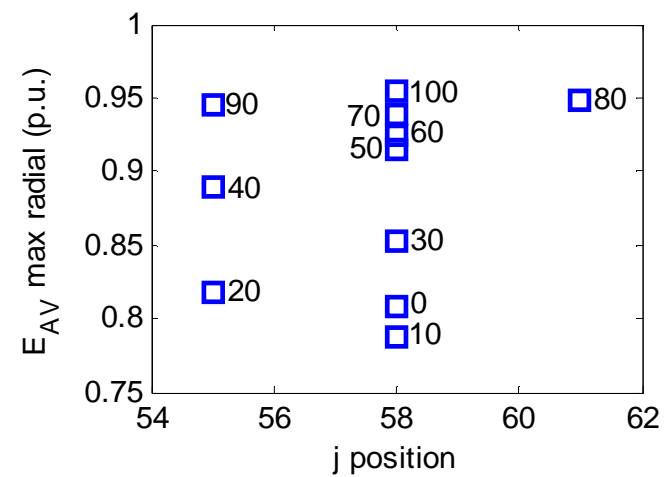

(b)

Figure 5. Peaks of emittance on the Axial (a) and radial (b) radiative profiles of the averaged frames for each measurement.

\subsection{Radiative power distribution}

In this analysis we consider ten different flame regions, these regions are separated in ten ranges of emittance starting from a minimum value of 0.55 (minimum value considered as part of the flame), figure 6 shows that the lower radiative areas are greater, while higher radiative areas and therefore the hottest ones, are smaller, becoming zero for lower air rates.

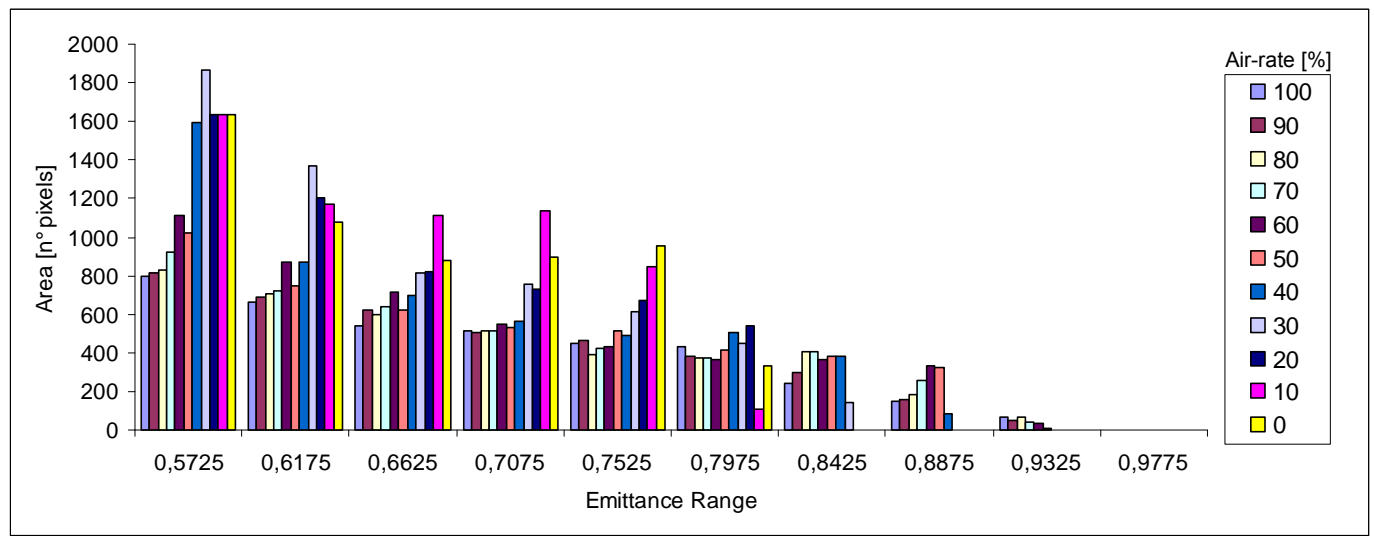

Figure 6. Radiative power distribution for different air rate conditions 


\subsection{Frequency analysis}

Figure 7.a shows the temporal variation of the third region for an air rate condition of $0 \%$, and figure 7.b shows the power spectral density of such signal. In order to obtain the spectral response the mean value of the temporal signal is subtracted from the original version of the area to avoid the DC value in the analysis.

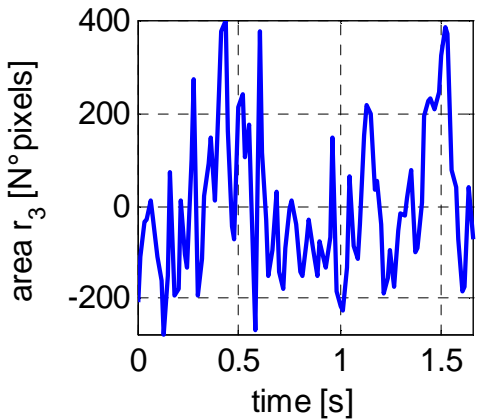

(a)

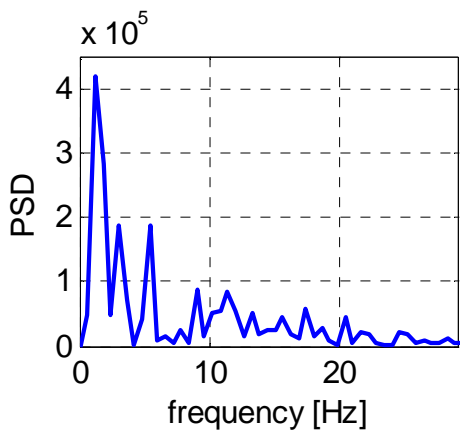

(b)

Figure 7. Temporal (a) and power spectral density (b) response of the area of region 3 for a 0[\%] of airrate condition.

As mentioned above, the flickering frequency is an important measure of the flame stability state. Thus, the power spectral density signals were calculated and then Eq. (1) was applied for the calculation of such frequencies. The results are shown in Fig. 8. It can be seen that for the three selected regions the flame becomes slightly more unstable for high air rate conditions, reaching a maximum of $10[\mathrm{~Hz}]$ for the first region. These results also show a parabolic pattern of the flickering frequency in function of the air rate and although they differ from other works $[7,8]$ it is a parameter that could predict the flame stability.

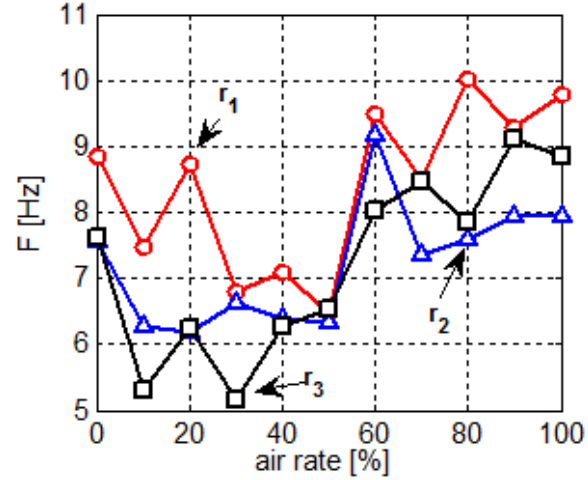

(a)

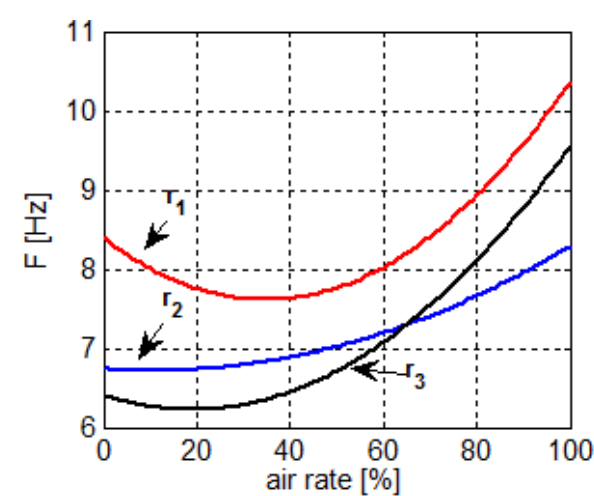

(b)

Figure 8. Flickering frequency for each region and air-rate condition, (a) samples (b) interpolated data.

\section{Conclusions}

In this work, a set of infrared flame images acquired from the combustion of LPG with air in a Bunsen burner were analyzed, each set of frames were acquired under different air-rate conditions. Summarizing, from the spatial analysis that the flame height and width varies, being smaller and wider for higher air rates and taller and thinner for the opposite conditions. The most radiative zones, and therefore the hottest, are concentrated in the central flame positions decreasing in intensity to the outside, and the peaks of emittance move axially to the burner when the air rate increases. The spatial power distribution is predominant in the less radiative regions and even zero for some of the higher ones. From the frequency analysis we see a relationship between the flickering frequencies calculated and the air rate conditions, which could be useful to predict the flame stability.

\section{Acknowledgments}

This work was partially supported by Programa de Financiamiento Basal grant PFB08024. Carlos Toro is also supported by a CONICYT Ph.D. Scholarship, Luis Arias is supported by FONDECYT Project 11110364 and Sergio Torres is supported by FONDECYT 1100522. 


\section{REFERENCES}

[1] Zárate L., "Study of the physical and geometrics characteristics of the forest fires flames". PhD. Thesis, Dept. of Chemical Engineering, Technical High School of Industrial Engineers of Barcelona, Polytechnich University of Cataluña, February, 2004.

[2] Muñoz M., Arnolds J., Casal J. and Planas E., "Analysis of geometrical and radiative characteristics of hydrocarbon pool fires". Combustion and Flame, Vol. 139, pp. 236-277, 2004. .

[3] Ballester J., García-Armingol T., "Diagnostic techniques for the monitoring and control of practical flames", Progress in Energy and Combustion Science, Vol. 36, pp. 375-411, 2010.

[4] Docquier N. and Candel S., "Combustion control and sensors: a review". Progress in Energy and Cmbustion Science, Vol. 28, pp.107-150, 2002.

[5] Arias L. et al, "On the spectral bands measurements for combustion monitoring", Combustion and Flame, Vol. 158, pp. 423-433, 2011.

[6] "Altair: Reference guide", FLIR systems, Advanced Thermal Solutions.

[7] Sun D., Lu G., Zhou H. and Yan Y., "Flame stability monitoring and characterization through digital imaging and spectral analysis", Measurement Science and Technology, Vol. 22, 2011.

[8] Huang Y., Yan Y., Lu G. and Reed A., "On-line flicker measurement of gaseous flames by image processing and spectral analysis", Measurement Science and Technology, Vol. 10, pp. 726-733, 1999. 